\title{
PROPTOSE EM CÃES E GATOS: ANÁLISE RETROSPECTIVA DE 64 CASOS
}

\section{(Proptose in dogs and cats: retrospective analyses of 64 medical records)}

\author{
BRANDÃO, C.V.S.'; RANZANI, J.J.T.; MARINHO, L.F.L.P; RODRIGUES, G.N. ${ }^{3}$; CREMONINI, D.N. ${ }^{3}$ \\ ${ }^{1}$ Professor Assistente Doutor do Departamento de Cirurgia e Anestesiologia Veterinárias da Faculdade de \\ Medicina Veterinária e Zootecnia da UNESP, Botucatu-SP; \\ ${ }^{2}$ Professor Titular Doutor do Departamento de Cirurgia e Anestesiologia Veterinárias da Faculdade de \\ Medicina Veterinária e Zootecnia da UNESP, Botucatu-SP; \\ ${ }^{3}$ Aluno de Pós-Graduacão da Faculdade de Medicina Veterinária e Zootecnia da UNESP, Botucatu-SP.
}

\begin{abstract}
RESUMO - Foi realizado um estudo retrospectivo envolvendo 45 cães e 19 gatos com proptose traumática do bulbo ocular. Foram estudados vários aspectos, tais como sexo, raça, idade, etiologia, tempo decorrente entre a lesão e admissão, seqüelas, além daqueles relacionados ao tratamento. O principal fator etiológico verificado entre cães foi a briga, enquanto na maioria dos gatos, a causa era desconhecida. Os cães adultos sem raça definida, seguidos pelo Pinscher, Poodle, Cocker Spaniel e Boxer foram os mais acometidos. As complicações mais comuns associadas à proptose foram ceratite ulcerativa, estrabismo, hifema, quemose, hiperemia, hemorragia de conjuntiva e periocular, ressecamento e opacidade da córnea, prolapso de terceira pálpebra, além de fratura nos ossos da face. Quinze cães e apenas 2 gatos avaliados foram submetidos ao reposicionamento do bulbo e tarsorrafia. Desses animais, somente 13 cães recuperaram a visão. Conclui-se que a precocidade na admissão dos cães influencia sobremaneira a obtenção de um prognóstico mais favorável, bem como na escolha do tratamento. Os gatos apresentam pior prognóstico em relação aos cães.
\end{abstract}

Palavras-chave: Cão, Gato, Proptose, Estrabismo, Estudo retrospectivo.

ABSTRACT - A retrospective study of 45 dogs and 19 cats with traumatic proptosis was performed. Sex, breed-specific, age, etiologic factors, time between trauma and admission, sequels and treatment were obtained for these animals. The principal etiologic factor in dogs was fight and in cats was unknown. Mongrel dogs, Pinscher, Cocker and Boxer were the most affected breeds. The common complications were corneal ulceration, strabismus, hyphema, chemosis, hyperemia, conjunctive hemorrhage, defects in the corneal epithelium, third eyelid prolapsed and facial bone fractures. Fifteen dogs and only two cats suffered repositioning followed by tarsorrhaphy. From these animals, only thirteen dogs recovered the eye vision. In conclusion, the precocity in the admission was important for a more favorable prognosis as much as the treatment choice. The cats presented worse prognosis when compared to dogs.

Key-words: Dog, Cat, Traumatic Proptosis, Strabismus, Retrospective study.

Correpondencia para: BRANDÃO, V.S. - Departamento de Cirurgia e Anestesiologia Veterinárias - Faculdade de Medicina Veterinária e Zootecnia da UNESP - Distrito de Rubião Júnior s/n - 18618-000 - Botucatu - SP - Email:valeriasb@fmvz.unesp.br. 


\section{Introdução}

A proptose é o deslocamento súbito do bulbo ocular, anteriormente, com simultâneo encarceramento pelas pálpebras atrás da região do equador (GELATT et al., 2003), podendo ser unilateral ou bilateral (BONAGURA, 2000). Sua etiologia inclui traumas, problemas dentários, corpos estranhos, neoplasias e predisposição racial (SMYTHE, 1956; COTTRELL, 1989; BONAGURA, 2000; MANDELL, 2000; GELLAT, 2003). Segundo GELLAT (2003), $50 \%$ dos cães afetados são braquicefálicos, podendo ocorrer após traumas leves (COTTRELL, 1989) devido a características anatômicas (WHELER et al., 2001). A ocorrência nas raças dolicocefálicas está associada com traumas severos. Nos gatos o espaço retrobulbar é limitado quando comparado aos cães (BONAGURA, 2000), sendo que nesses, geralmente, a proptose está associada a traumas severos, fraturas faciais, hemorragia orbitária (COTTRELL, 1989), hifema, perfuração corneana, lesão uveal, midríase paralítica, avulsão de três ou mais músculos extra-oculares, bem como lesão do nervo óptico, condições estas que admitem prognóstico desfavorável (GILGER et al., 1995).

As proptoses requerem assistência emergencial, por proporcionar oclusão das veias vorticosas e ciliares pelas pálpebras, resultando em estase venosa e glaucoma congestivo, além de promover necrose corneana, ceratites por exposição, irites, coriorretinites, descolamento da retina, luxação do cristalino, avulsão óptica e, na maioria das vezes, estrabismo (SLATTER, 1990). A reintrodução do bulbo ocular é procedimento obrigatório nas proptoses, exceto nos casos associados à extrusão ou destruição de suas estruturas internas (BISTNER, 1998), bem como naqueles com ruptura da maioria dos músculos extra-oculares, presença de tecido necrótico ou altamente infectado, pupila dilatada ou outras complicações irreversíveis; para estes, o tratamento de escolha é a enucleação, porém em alguns casos, há a possibilidade de manutenção do bulbo ocular com propósitos estéticos (GELATT, 2003).
GILGER et al. (1995) observaram que $27 \%$ dos animais apresentaram estrabismo após o reposicionamento cirúrgico do globo com proptose, sendo que, decorridos 26 dias, este índice elevava-se para $36 \%$. Segundo os autores, os músculos mais freqüentemente acometidos por roturas foram o reto medial, reto ventral e oblíquo ventral. A grande incidência de rotura do músculo reto medial e reto ventral decorre de sua extensão limitada e localização, anterior aos demais músculos extra-oculares (GILGER et al., 1995), resultando em estrabismos divergentes, superiores ou cicatriciais causados por fibrose pósoperatória (BISTNER, 1998). Quando a miorrafia não é possível nas rupturas da musculatura extra-ocular, preconiza-se a adoção de métodos auxiliares, tais como, a reconstrução utilizando-se cápsula de Tenon (BERENS, 1937), membrana amniótica, peritônio (DUNLAP, 1968), músculos autógenos homotópico (SILVA, 1983), e cápsula renal (MAMEDE, 2003). Seqüelas de proptose incluem perda da visão, estrabismo, lagoftalmia, déficit sensitivo da córnea, ceratoconjuntivite seca, ceratites por exposição, glaucoma e phthisis bulbi (GELATT, 2003). Portanto, o escopo do estudo é informar e auxiliar os profissionais, a despeito da magnitude, comportamento e tratamento da proptose em casos atendidos no hospital veterinário da Unesp - FMVZ Campus de Botucatu.

\section{Material e Método}

Um estudo retrospectivo envolvendo 45 cães e 19 gatos que apresentaram proptose do bulbo ocular atendidos no Hospital Veterinário foi realizado no período de agosto de 1998 a agosto de 2003.

Todos os casos clínicos foram analisados segundo espécie, raça, idade, sexo, etiologia, olho acometido, tempo decorrente entre a lesão e admissão, lesões e seqüelas associadas à enfermidade. $O$ tratamento utilizado também foi estudado e classificado de acordo com as diferentes condutas clínico-cirúrgicas, ou seja, enucleação ou reposicionamento seguido por tarsorrafia. 


\section{Resultados}

Os cães acometidos por proptose apresentaram a seguinte distribuição com relação às raças: sem raça definida $(53,33 \%)$, Pinscher (13,33\%) Poodle e Cocker Spaniel Inglês (6,67\%), Boxer, Pequinês e Dachshund (4,44\%), Pug, Shi-Tzu, Chihuahua (2,22\%). Dentre os gatos, houve uma incidência dos animais de pêlo curto sem raça definida $(84,2 \%)$ em relação aos Siameses (15,8\%). A idade média dos cães foi de 4,4 anos, variando entre um mês e 12 anos; nos gatos, foi de um ano, variando entre um mês e 5 anos. A relação entre machos e fêmeas na espécie canina foi $28 / 17$ e na felina $13 / 6$.

A despeito da etiologia da proptose nos cães, $42 \%$ dos casos estavam relacionados a brigas, enquanto que os animais atropelados e os de causa não estabelecida somaram $29 \%$ cada grupo. Já em relação aos gatos, 37\% tiveram envolvimento em brigas, apenas $10 \%$ em atropelamento e $53 \%$ origem desconhecida.

O envolvimento do olho direito foi verificado em $53 \%$ cães e em $42 \%$ dos gatos. O tempo para admissão dos cães no Hospital Veterinário variou de uma hora a 5 dias pós-trauma, sendo que $13 \%$ foram atendidos nas primeiras duas horas, $18 \%$ entre duas e seis horas, $51 \%$ entre seis e 24 horas e o restante compreendido por $18 \%, 24$ horas após a ocorrência do trauma. Apenas $5 \%$ dos gatos foram admitidos até 12 horas pós-trauma; o restante, representado por $47,5 \%$ entre 12 e 24 horas e a mesma porcentagem após 72 horas, perfazendo uma variação de oito horas a 8 dias.

Nos cães, as complicações mais comuns associadas à proptose foram ceratite ulcerativa, estrabismo, hifema, quemose, hiperemia, hemorragia de conjuntiva e periocular, ressecamento e opacidade da córnea além de fratura nos ossos da face. Os gatos apresentaram ainda prolapso de terceira pálpebra. Lagoftalmia, sinéquia anterior, ceratoconjuntivite seca, neovascularização de córnea, blefaroespasmo e fotofobia foram observados em ambas as espécies, porém, em menores proporções. Na maioria dos animais notou-se avulsão de mais de um músculos extraoculares, verificados ao exame clínico e à enucleação.
Não foi possível a avaliação do segmento posterior e de suas estruturas na maioria dos animais, devido à dificuldade de visualização proporcionada pelo hifema e outras complicações. Vinte e oito cães e 19 gatos foram considerados cegos no momento da admissão. Os animais foram submetidos a enucleação ou reposicionamento seguido de tarsorrafia. A enucleação foi realizada em 30 cães e 17 gatos; os demais foram submetidos ao reposicionamento seguido de tarsorrafia. Treze cães $(28,8 \%)$ recuperaram a visão, o que não foi observado nos gatos, pois nenhum recuperou a visão.

\section{Discussão e Conclusão}

O presente estudo revelou uma alta incidência de proptose em cães e gatos machos, similar ao relatado em estudo realizado por GILGER et al. (1995), em função das características comportamentais que viabilizam o acesso à rua, justificando essa maior incidência.

A despeito da etiologia em cães, $42 \%$ dos casos eram resultados de brigas, semelhante aos $41 \%$ descrito por GILGER et al. (1995), sendo essa a principal causa relatada por outros autores (MANDELL, 2000; GELLAT, 2003). Em gatos, $53 \%$ dos casos eram de etiologia desconhecida, decorrente, provavelmente, do hábito felino de saídas constantes à rua, dificultando a vigilância dos mesmos.

O período entre o momento do trauma e admissão dos animais no Hospital Veterinário não teve correlação com o retorno da visão dos cães, segundo estudo realizado por GILGER et al. (1995). Segundo o autor, o tratamento precoce pode ajudar a resolver a inflamação e edema. Já com relação aos gatos, segundo os autores, o tempo de admissão influenciou sobremaneira e isto pode ter contribuído para o fato de que nenhum gato recuperou a visão no olho afetado. No presente estudo, a precocidade na admissão influenciou tanto a escolha do tratamento quanto a evolução e prognóstico da enfermidade, visto que os animais que foram atendidos rapidamente, apresentaram taxas maiores na recuperação da visão. 
Um aspecto que deve ser destacado para a melhora no prognóstico desta afecção é a conscientização dos proprietários com relação à procura de serviço veterinário especializado o mais rápido possível, além de maior controle dos seus cães e gatos em sua residência.

Com relação ao olho acometido, não houve diferença significativa entre os lados. GILGER et al. (1995) obtiveram resultados bem diferentes expressados por $41 \%$ e $72,3 \%$.

Não foi possível a avaliação da câmara vítrea e suas estruturas na maioria dos animais, devido às complicações que proporcionaram dificuldade de visualização, fato esse citado pela literatura veterinária (SLATTER, 1990). Em três cães foi possível a visualização do segmento ocular posterior; este encontrava-se normal, sendo que após o reposicionamento, estes animais apresentaram retorno da visão. Já nos gatos, apenas dois possibilitaram a visualização; destes, somente um animal apresentava-se normal, no entanto, este não apresentou uma boa evolução. GILGER et al. (1995) também descreveram que o único cão em que foi possível a visualização do segmento posterior aparentemente normal, demonstrou retorno da visão após o reposicionamento seguido de tarsorrafia.

Dentre as complicações, o estrabismo, principalmente divergente resultante da avulsão dos músculos extra-oculares, é o mais comum (BISTNER, 1998). GILGER et al. (1995) relataram o mesmo fato em seu estudo com proptose ocular traumática; salientaram ainda que cerca de $27 \%$ dos animais apresentaram estrabismo após reposicionamento cirúrgico, sendo que decorridos 26 dias, o percentual aumentava para $36 \%$. No presente estudo, notou-se que os animais com avulsão de vários músculos extra-oculares na sua grande maioria não recuperaram a visão, provavelmente pelo papel fundamental destes tecidos na preservação do nervo óptico e vascularização da retina e outras estruturas oculares importantes. Segundo os autores, os animais que tiveram avulsão de três ou mais músculos extra-oculares perderam a função visual.

Independentemente do tratamento, apenas 13 cães e nenhum gato recuperaram a visão. Isto significa $28 \%$ do total de cães versus $27 \%$ do estudo realizado por GILGER et al. (1995).
O presente estudo, reforça a piora do prognóstico nos casos associados a fraturas faciais, lesão do nervo óptico e avulsão de vários músculos extra-oculares; sendo que nos felinos, o prognóstico quase sempre é desfavorável em decorrência da sua conformação anatômica e constante associação a complicações graves. Dentre os animais atendidos, observou-se maior incidência nos animais sem raça definida; seguidos nos cães pelas raças pinscher, poodle e cocker.

Tanto no cão quanto no gato, verificou-se maior incidência em machos; entretanto, os gatos apresentaram-se numa idade média mais precoce (1 ano) em relação aos cães (4 anos). Conclui-se que a precocidade na admissão influencia a obtenção de um prognóstico mais favorável, bem como a escolha do tratamento.

\section{REFERÊNCIAS}

BERENS, C. Tenon's capsule transplantation in surgery of the ocular muscles, with especial referency to postoperative deviations with adhesions between the muscles and eyeball. Transplantation American Ophthalmology Society, Rochester, v.35, n.1, p.173-183, 1937.

BISTNER, S.I. Emergências e traumatismos oculares. In: SLATTER, D. Manual de cirurgia de pequenos animais. 2 ed. São Paulo: Manole, 1998. p.1522-1539.

BONAGURA, J.D. Ocular emergencies. In: KIRK \& BONAGURA, J.D. Kirk's current veterinary therapy XIII small animal practice. Philadelphia: W. B. Saunders, 2000. 1090 p.

COTTRELL, B. Abnormal appearance. In: PEIFFER, R.L. Small animal ophthalmology: a problem oriented approach. Philadelphia: W. B. Saunders, 1989, p.135-138.

DUNLAP, E. A. Plastic implants in muscle surgery, plastic materials in the management of extra-ocular motility restrictions. Archives of Ophthalmology, Chicago, v.80, n.2, p.249-57, 1968.

GELATT, K.N. Doenças e cirurgia da órbita do cão. In: Manual de oftalmologia veterinária. 3. ed. São Paulo: Manole, 2003. p.39-42.

GILGER, B.C.; HAMILTON, H.L.; WILKIE, D.A.; WOERDT, A.; MCLAUGHIN, S.A.; WHITLEY, D.R. Traumatic ocular proptose in dogs and cats: 84 cases (1980-1993). Journal of American Veterinary Medical Association, Chicago, v.206, n.8, p.1186-90, 1995. 
MAMEDE, F.V. Emprego da mitomicina C, como agente antifibrótico, na mioplastia do reto medial do olho de coelhos (Oryctolagus cuniculus, Linnaeus, 1958) pela cápsula renal do eqüino (Equus caballus, Linnaeus, 1958). Botucatu, 2003. 57 p. Dissertação (Mestrado em clínica) - Curso de Medicina Veterinária, Universidade Estadual Paulista. MANDELL, D.C. Ophthalmic emergencies. Clinical techniques small animal practice. Philadelphia, v.15, n.2, 2000, p.94-95.

SILVA, L.H. Transplante autólogo, homotopico, livre, de músculo ocular extrínseco com e sem uso de antiadesivo: estudo experimental. Ribeirão Preto, 1983. 56 p. Dissertação (Mestrado em Oftalmologia) - Curso de Medicina, Universidade de São Paulo.

Recebido para publicação: 24/01/2005 Aprovado:
SLATTER, D. Ocular emergencies. In: SLATTER, $D$. Fundamentals of veterinary ophthalmology. 2.ed. Philadelphia: W.B. Saunders, 1990. p.537540.

SMYTHE, R. H. The eyeball and its surroundings. In: ___. Veterinary ophthalmology. London: Baillire Tindall and Cox, 1956. p. 86-89.

WHELER, C.L.; GRAHN, B.H; POCKNELL, A.M. Unilateral proptosis and orbital cellulitis in eight african hedgehogs (atelerix albiventris). Journal of Zoo and Wildlife Medicine, Lawrence, v.32, n.2, p.236-237, 2001. 